\title{
NAFLD progression is related with plasma pro- oxidative biomarkers rather than liver tissue- measured nitrogen metabolism biomarkers in population with obesity and metabolic syndrome
}

\section{Juan Antonio Suárez-Cuenca ( $\nabla$ suarej05@gmail.com )}

Centro Médico Nacional 20 de Noviembre https://orcid.org/0000-0002-2098-5658

Gabriela Alexandra Domínguez-Pérez

Instituto de Seguridad y Servicios Sociales de los Trabajadores del Estado

Rolando Efraín Hernández-Muñóz

Instituto de Seguridad y Servicios Sociales de los Trabajadores del Estado

Alejandro Hernández-Patricio

Instituto de Seguridad y Servicios Sociales de los Trabajadores del Estado

Eduardo Vera-Gómez

Instituto de Seguridad y Servicios Sociales de los Trabajadores del Estado

Juan Ariel Gutiérrez-Buendía

Instituto de Seguridad y Servicios Sociales de los Trabajadores del Estado

Ollin Cázares-García

Instituto de Seguridad y Servicios Sociales de los Trabajadores del Estado

Moisés Salamanca-García

Instituto de Seguridad y Servicios Sociales de los Trabajadores del Estado

Moisés Ortíz-Fernández

Instituto de Seguridad y Servicios Sociales de los Trabajadores del Estado

Jesús Montoya-Ramírez

Instituto de Seguridad y Servicios Sociales de los Trabajadores del Estado

Omar Felipe Gaytán-Fuentes

Instituto de Seguridad y Servicios Sociales de los Trabajadores del Estado

Ricardo Blas-Azotla

Instituto de Seguridad y Servicios Sociales de los Trabajadores del Estado

Alberto Melchor-López

Instituto de Seguridad y Servicios Sociales de los Trabajadores del Estado

Rebeca Pérez-Cabeza de Vaca

Instituto de Seguridad y Servicios Sociales de los Trabajadores del Estado

Paul Mondragón-Terán

Instituto de Seguridad y Servicios Sociales de los Trabajadores del Estado 


\section{Research article}

Keywords: Pro-oxidative biomarkers, ammonium, nitrites, arginine, citrulline, NAFLD progression, obesity, metabolic phenotype, metabolic syndrome

Posted Date: January 20th, 2020

DOl: https://doi.org/10.21203/rs.2.21311/v1

License: (c) (i) This work is licensed under a Creative Commons Attribution 4.0 International License. Read Full License 


\section{Abstract}

\section{Background.}

The metabolic syndrome (MS) is associated with an increased production of nitrogen metabolites and elevated oxidative stress, which favor the development and progression of non-alcoholic fatty liver disease (NAFLD). Subjects with the phenotype known as metabolically unhealthy obese (MUO) meet most of the MS cardiometabolic risk criteria and show a higher prevalence of NAFLD and fibrosis progression risk, compared to the so-known as metabolically healthy obese (MHO). The present study aimed to explore whether liver-specific biomarkers of nitrogen metabolism and oxidative stress, measured in both plasma and in liver tissue, may relate to NAFLD severity and/or metabolic phenotype.

Methods.

This observational, cross-sectional study included candidates for bariatric surgery with biopsy-proven NAFLD diagnosis and staging. For comparison, the study population was divided according to NAFLD progression (steatohepatitis F0-F1 vs F2-F4) and metabolic phenotype (MHO vs MUO, based on the MS criteria). Hepatic and plasma concentrations of nitrogen metabolites and oxidative stress biomarkers were determined by enzymatic kinetics assays, ELISA and Griess reaction.

Results.

The study population consisted of 28 patients with obesity and higher prevalence of dyslipidemia, diabetes mellitus and hypertension. NAFLD progression was related with the metabolic phenotype. According to plasma biomarkers, MUO phenotype was related to higher cardiometabolic risk; while NAFLD severity progression was related to higher $\mathrm{Hb}$ A1c and triglycerides. Elevated hepatic concentrations of ammonium, nitrites, arginine and citrulline were found in MUO phenotype, but only higher plasma concentration of MDA was found as specifically related to NAFLD progression.

\section{Conclusions.}

Circulating biomarkers of redox state were selectively related with NAFLD progression, supporting prognostic and therapeutic potential target. Hepatic concentrations of nitrogen metabolism biomarkers may be more related to cardiometabolic risk.

\section{Background}

Non-alcoholic Fatty Liver Disease (NAFLD) affects about 30\% of the population worldwide and Mexican population owns several risk factors for NAFLD development, whereas prevalence has been estimated as higher than 50\% (Bernal R, 2019). NAFLD has become a public health problem due to potential liver damage progression including fibrosis, cirrhosis or adenocarcinoma (Angulo P, 2012).; and cost for therapy may reach 7,804 USD a new NAFLD diagnosis and 3,789 USD for long-term management (Allen A, 2018). 
NAFLD progression is influenced by local noxious environmental mediators such as oxidative stress and nitrogen metabolites (Drake IM, et al., 1996); as well as metabolic factors like obesity, diabetes mellitus and/or dyslipidemia. In fact, NAFLD is particularly frequent in population with metabolic syndrome (MS); while obese individuals with MS are particularly susceptible for abnormal lipid accumulation in different tissues (Chen G, et al., 2016).

Currently, a clear pathophysiological characterization of local noxious mediators and metabolic risk factors during NAFLD severity progression is lacking. Likewise, whether hepatic damage is reflected by plasma biomarkers in patients with NAFLD and MS is unknown.

The present study evaluated the activity of enzymes related to oxidative stress, nitrogen metabolism, both in plasma and in liver tissue, which may identify potential targets for prevention and/or prognosis of NAFLD severity progression in population with MS.

\section{Methods}

Observational, cross-sectional, analytical and comparative study, performed at "Centro Médico Nacional "20 de Noviembre", ISSSTE, a third level referral medical center in Mexico City, designed to study whether liver specific biomarkers of nitrogen metabolism and oxidative stress may relate to NAFLD severity and/or metabolic phenotype.

This project was registered (ID 500.2017) and authorized by Institutional Ethics and Biosafety Review Boards and complies with recommendations from Declaration of Helsinki. All participants signed informed consent.

\section{Study population}

Study population was recruited from 2016 to 2018 at the Department of Bariatric Surgery. Subjects with obesity, candidates for bariatric surgery (body mass index (BMI) >40 or BMI>35 with co-morbidities), older than 18 years old, with and without MS and. Subjects were eliminated if they consumed drugs with potential effect on oxidative stress or nitrogen metabolism mediators, or were exposed to any hepatotoxic condition (alcohol, drugs, hepatitis virus, cholestasis, etc). All patients had psychological and endocrine profile assessments.

\section{Demographic, Anthropometric Data and Samples Collection}

Age, sex, BMI, waist circumference and the blood pressure were obtained during clinical history and physical exam before bariatric surgery. A sample of peripheral venous blood $(10 \mathrm{~mL})$ was obtained and centrifuged to obtain plasma, which was frozen at $-80^{\circ} \mathrm{C}$ until final analysis. Liver biopsy was performed during bariatric surgery, in those cases with previous biochemical or ultrasound evidence suggestive of NAFLD; or at surgeon discretion, based on macroscopic liver tissue changes.

\section{Plasma Biomarkers}


Quantification of plasma glucose, lipid profile and $\mathrm{Hb}_{\mathrm{A} 1 \mathrm{c}}$ was evaluated through routine automatized nephelometric laboratory assays. Plasma concentration of Malonildialdehyde (MDA) and of nitric oxide, were determined by Colorimetric/Fluorometric Assay Kit, BioVision Incorporated, CA, USA; and Nitric Oxide Colorimetric Assay Kit, BioVision Incorporated, CA, USA; respectively, following provider's instructions.

\section{Hepatic Biomarkers}

A liver sample of approximately $0.5-1.0 \mathrm{~cm}^{3}$ was obtained during the surgical procedure. Then, $200 \mathrm{mg}$ of liver tissue was deproteinized in $1 \mathrm{~N}$ perchloric acid, neutralized and the following analytes were determined in the acid extracts: nitrite (Griess reaction), citrulline (Green LC, et al, 1982; Cerotti G, et al, 1983) and pyruvate (Gutiérrez JA, et al., 1999). Additionally, the amount of MDA was measured by its reaction to thiobarbituric acid (Hernández-Muñoz R, et al, 1987). The activities of the arginase and Ornithine Transcarbamylase-transferase enzymes were also determined by previously described techniques (Xiong XF et al., 1989).

\section{NAFLD severity progression}

A piece of liver tissue was fixed in $4 \%$ paraformaldehyde, paraffin-embedded and slides were stained with H\&E and Masson's trichrome stain. Histological assessment of liver fibrosis progression was performed by an experienced pathologist, based on Brunt and Kleiner classification ( 0 , no fibrosis; 1 , perisinusoidal or periportal; 2, Perisinusoidal and portal/periportal; 3, Bridging fibrosis; 4, Cirrhosis. (Kleiner DE, 2005; Brunt EM, 2011). Only for analytic purposes in this study, low liver fibrosis was considered when stages 01 , whereas advanced liver fibrosis was considered with stages 2-4.

\section{Metabolic risk phenotype and subgroups}

MS was defined according to the US Adult Treatment Panel-III criteria (NCEP, 2001), meeting at least 3 of the following 5 criteria: 1) Fasting plasma glucose above $100 \mathrm{mg} / \mathrm{dl}$; 2) Plasma triglyceride level $\geq 150$ $\mathrm{mg} / \mathrm{dl}$; 3) Plasma high-density lipoprotein (HDL) level for women $\leq 50 \mathrm{mg} / \mathrm{dl}$, or for men $\leq 40 \mathrm{mg} / \mathrm{dl} ; 4$ ) Blood pressure $\geq 130 / 85 \mathrm{mmHg}$ and 5) Waist circumference for women $>88 \mathrm{~cm}$, for men $>102 \mathrm{~cm}$.

Patients without MS were considered as metabolically healthy obese (MHO) patients. Likewise, the patients meeting MS criteria were considered as metabolically unhealthy obese (MUO) patients. In order to perform a systematic analysis, we subdivided the study population according to the metabolic phenotype and the hepatic fibrosis progression, as follows: group 1: MHO phenotype with liver fibrosis stages 0 to 1 (low fibrosis); group 2: MUO with liver fibrosis 0 to 1(low fibrosis); and group 3: MUO with liver fibrosis $>1$ (advanced fibrosis).

\section{Statistical analysis}

Due to methodological strategy of the study, sample size was calculated considering $95 \% \mathrm{Cl}$ alpha $=0.05$, alpha $=0.05$, adjusted by the total number of patients who meet selection criteria, with an estimated beta power of 0.74 . Data were expressed in median (IQR) or $n(\%)$ according to their nature. Wilcoxon rank-sum 
was performed for median comparison, while analysis of variance (ANOVA) was applied for qualitative data (one-way; $p<0.05$ was considered statistically significant). Data were analyzed using SPSS v.25 (IBM Corp., Armonk, NY, USA).

\section{Results}

The study population consisted of 28 obese patients (female, 63.3\%), candidates for bariatric surgery, characterized by higher prevalence of dyslipidemia, diabetes and hypertension, whose characteristics are shown in Table 1.

Table 1. Characteristics of the Study Population $(n=28)$

\begin{tabular}{|c|c|c|c|}
\hline & All $(n=28)$ & $\mathrm{MHO}(\mathrm{n}=9)$ & MUO $(n=19)$ \\
\hline Age & $42(39,48.5)$ & $45(39.75,48.7)$ & $41(38.5,46.5)$ \\
\hline Female, n(\%) & $18(64.3)$ & $6(66)$ & $12(63)$ \\
\hline BMI (kg/m2) & $42.8(38.04,50.2)$ & $39.65(37.37,43.6)$ & $44.9(39.3,51.46)$ \\
\hline WC (cm) & $134(125.5,147.3)$ & $128(124,147.3)$ & $138(129,147.8)$ \\
\hline SBP $(\mathrm{mmHg})$ & $130(110,130.5)$ & $117.5(110,131)$ & $130(115,145)$ \\
\hline $\mathrm{DBP}(\mathrm{mmHg})$ & $80(74.5,90)$ & $80(72.25,87.5)$ & $80(75,90)$ \\
\hline FPG & $101(89,113.5)$ & $88.5(84.25,98.5)$ & $110(98,117)$ \\
\hline $\mathrm{Hb}_{\mathrm{A} 1 \mathrm{c}}(\%)$ & $6.1(5.75,6.45)$ & $5.9(5.65,6.1)$ & $6.4(5.85,7.1)$ \\
\hline $\mathrm{TG}(\mathrm{mg} / \mathrm{dl})$ & $147(83,219.5)$ & $120(78,147.8)$ & $200(94.5,241.5)$ \\
\hline HDLc (mg/dl) & $41(33.6,51)$ & $41(35.93,47.95)$ & $35.3(30.2,55.75)$ \\
\hline
\end{tabular}

Patients were further grouped according to cardiometabolic risk and biopsy-proven liver fibrosis progression (Table 2 and Fig. 1, respectively). A subgroup of MHO with advanced liver fibrosis was unable to integrate, due to the very low number of patients. 
Table 2. Metabolic Profile and Liver Fibrosis Progression $(n=28)$

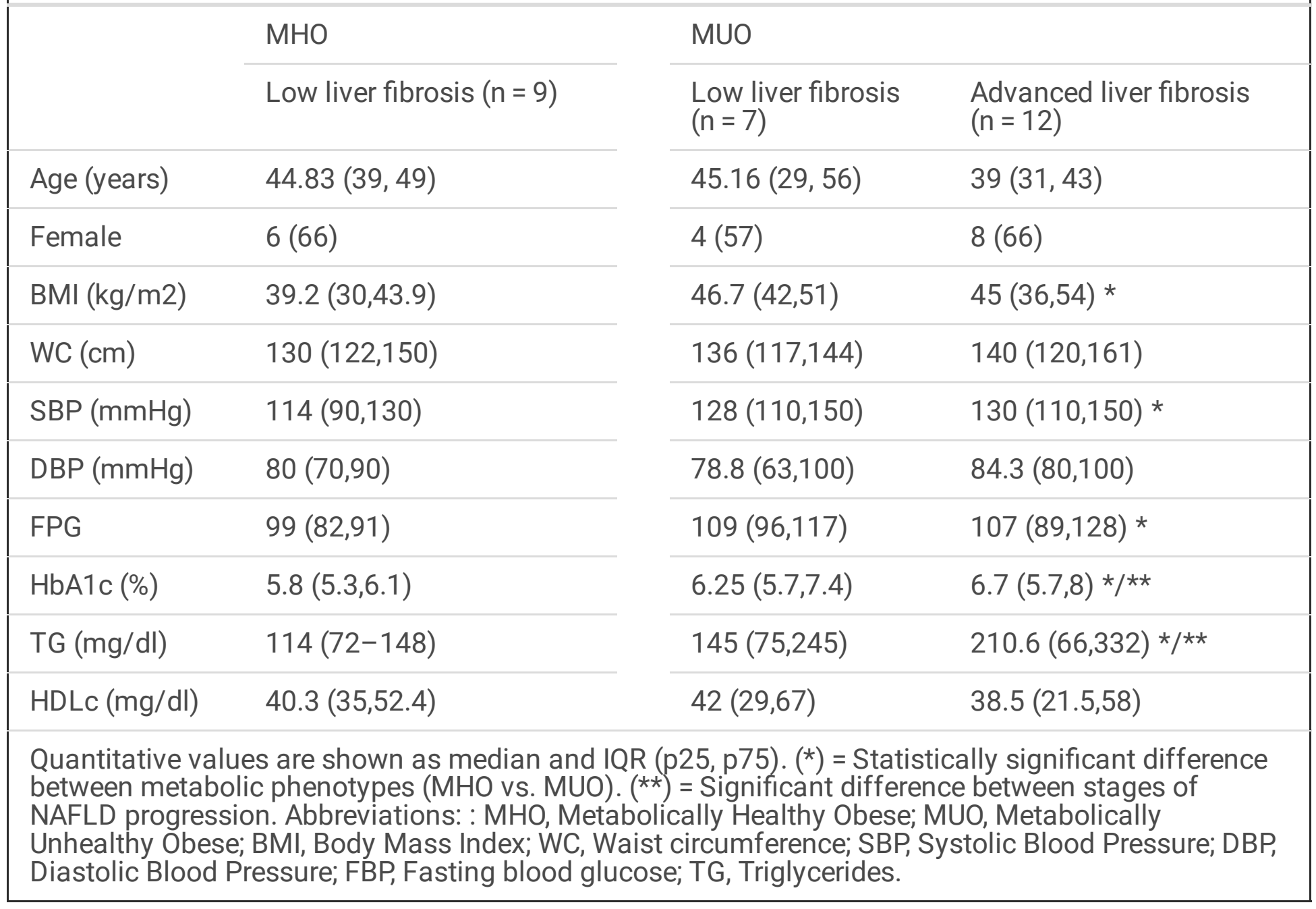

The MUO phenotype was characterized by higher BMI, SBP, plasma FPG, HbA1c and TGs; while the progressive liver fibrosis was related with higher plasma HbA1c and TG (Table 1).

Liver concentration of ammonium, nitrites, arginine and citrulline are significantly elevated in MUO phenotype, regardless of NAFLD condition. Ornithine concentration was not related to progression of liver fibrosis (Fig. 1).

On the other hand, higher plasma concentration of MDA was found as specifically related to the progression of NAFLD, regardless the metabolic phenotype (Fig. 2); while plasma nitric oxide did not show significant variation.

\section{Discussion}

Our main finding was that circulating pro-oxidative mediator MDA was selectively related with NAFLD progression; while hepatic concentrations of nitrogen metabolism biomarkers were more associated with the cardiometabolic phenotype. 
Consistently, the study from Gutiérrez-Grobe, et. al. revealed that patients with MUO phenotype showed a higher prevalence of liver fibrosis in comparison with $\mathrm{MHO}$ phenotype between Mexican population (Gutiérrez-Grobe, et. al. 2017).

Likewise, oxidative stress has been concomitantly related with obesity and NAFLD. Obese patients show lower plasma concentration of glutathione peroxidase (Leghi GE, et al., 2015) while oxidative stress is considered a key pathological event during NAFLD development and progression (Spahis S, 2017). In this context, antioxidants therapy combined with hypocaloric diet may induce NAFLD regression (Aller R, 2015). In addition, several evidences support the role of oxidative stress in NAFLD progression: 1) increase in ROS may promote hepatocyte release of pro-inflammatory cytokines (Liu J, 2016) and trigger transition from steatosis to steatohepatitis (Sodki K, et. al., 2015); 2) lipid peroxidation, as reflected by MDA, may be related with depletion of n-3 long-chain poly unsaturated fatty acids and impairment of fatty acid oxidation and triacylglycerol metabolism (Videla LA, 2004); 3) a recent study shows that oxidative stress in the liver tissue was related to increased lipotoxicity and steatosis exacerbation in humans (Masarone M, 2018).

In our study, circulating MDA was more associated to NAFLD progression than hepatic mediators of metabolic damage. This observation is consistent with previous study where bone marrow fat content correlated with hepatic fat content in children with NAFLD (Yu NY, 2017); suggesting that metabolic disorders in other organs or systems may influence local hepatic metabolism, which would be particularly relevant for NAFLD development.

We also found that nitrogen metabolism biomarkers within the hepatic tissue were mainly related with cardiometabolic phenotype, more than NAFLD progression; which may have several possible explanations. 1) MUO phenotype shows a higher activation of the NLP3-inflammasome related molecule in macrophages in adipose tissue, as compared with $\mathrm{MHO}$. Such difference may favor pro-inflammatory responses (Stienstra R, et.al. 2013), along the ability of NLP3 to interact with protein PYCARD/ASC and promote local inflammation and apoptosis, which may be involved in NAFLD progression (Henao-Mejia J, et.al, 2012); 2) liver metabolism disorders and lipid accumulation in hepatocytes are strongly linked to insulin resistance and components of MS, which are cumulative in the MUO phenotype. Indeed, we observed that MUO phenotype as well as NAFLD progression correlated with increasing values of Hba1c, but this last marker lacked liver selectivity.

Furthermore, the finding that plasma oxidative biomarkers are related with MS in population with NAFLD acquires relevance because of the higher prevalence of NAFLD between patients with MS combined with higher cardiovascular risk (Hoening ME, et al., 2010). The pro-oxidant biomarkers associated with NAFLD progression in the present study have been also described as pro-inflammatory and pro-atherogenic mediators; then, representing a potential link between NAFLD and cardiovascular disease (Dowman JK, 2010).

Recently, the relation between NAFLD and cardiovascular disease has been increasingly recognized. Heart diseases are the most common cause of mortality in individuals with NAFLD (Adams, 2005), larger 
than the number of liver-related deaths; whereas the estimated 10-year risk score is increased in individuals with NAFLD, and multivariable regression analyses demonstrate up to a 3.4-fold increase in cardiovascular risk for advanced stages of hepatic steatosis. Consistently, the group with MUO phenotype from our study population shared several components of MS and higher cardiovascular risk.

We acknowledge several limitations in the present study: First, a cross-sectional study could not objectively evaluate the time-course prospective evolution NAFLD progression and related biomarkers; therefore, a causal role of the proposed study biomarkers deserves careful interpretation. Second, small sample size; which may be compensated by the methodological design of a concomitant study of liver and plasma tissue biomarkers. This is relevant, since liver biopsy is the gold standard for the diagnosis of fibrosis and precise classification of NALFD, whereas only few studies have been able to do so. Despite the small sample size, we were able to obtain significant results in the biomarkers.

Finally, our study suggests that patients with MS and NAFLD should not be treated equally, given their corresponding association with either cardiovascular risk or pro-oxidant status and NAFLD progression, respectively.

\section{Conclusion}

Circulating biomarkers of redox state are selectively related with NAFLD progression supporting prognostic and therapeutic potential target; while hepatic concentrations of nitrogen metabolism biomarkers may be explained by the cardiometabolic risk phenotype.

\section{Abbreviations}

MS. Metabolic Syndrome

NAFLD. Non-alcoholic fatty liver disease

MUO. Metabolically unhealthy obese

MHO. Metabolically healthy obese

ELISA. Enzyme-Linked Inmmunosorbent Assay

HbA1c. Hemoglobin A1c

ISSSTE. "Instituto de Seguridad y Servicio Social para los Trabajadores del Estado"

BMl. Body mass index

MDA. 3,4-Methylenedioxyamphetamine

NCEP: National Cholesterol Education Program 
ANOVA. Analysis of Variance

IQR: Interquartile Range

WC. Waist circumference

SBP. Systolic blood pressure

DBP. Diastolic blood pressure

FPG. Fasting blood glucose

TG. Triglycerides

HDLc.. High Density Lipoprotein Cholesterol

LF. Low fibrosis

HF. High fibrosis

\section{Declarations}

Ethics approval and consent to participate. This project was registered (ID 500.2017) and authorized by Institutional Ethics and Biosafety in Research Review Boards and complies with recommendations from Declaration of Helsinki.

Consent for publication. Not applicable.

Competing interests. The authors declare that they have no competing interests.

Availability of data and materials. The datasets generated and/or analyzed during the current study are not publicly available due to institutional data use guidelines, but are available from the authors on reasonable request.

Funding. The authors state that this study was partially supported by grant from Consejo Nacional para la Ciencia y Tecnología (CONACYT) and Programa E-015 Investigación y Desarrollo Tecnológico en Salud (ISSSTE).

Authors contributions. The authors certify that each author participated sufficiently in the study, JAS-C designed the study, discussed and interpreted the data and drafted the manuscript. GAD-P collected the liver biopsies and the data, carried out the colorimetric assay techniques and analysis, discussed and interpreted the data and drafted the manuscript. REH-M conceived the study, carried out the colorimetric assay techniques and analysis, discussed and interpreted the data and drafted the manuscript. AH-P and EV-G carried out the colorimetric assays and analysis, discussed and interpreted the data and drafted the manuscript. JAG-B and CG-G collected the liver biopsies and the data, discussed and interpreted the data 
and drafted the manuscript. MS-G designed the study, performed the histopathological grading of steatosis and fibrosis, discussed and interpreted the data and drafted the manuscript. JM-R, MOR-F, ORG$\mathrm{F}$ and RB-A designed the study, performed the surgical interventions and obtained the liver biopsies, discussed and interpreted the data and drafted the manuscript. AM-L, RPC-V and PM-T designed the study, discussed and interpreted the data and drafted the manuscript. All authors have agreed to be accountable for all aspects of the work. All authors have read and approved the final manuscript.

Acknowledgements. The authors acknowledge Miss Cristina Rubio-Rubio for her administrative assistance.

\section{References}

Adams LA, Lymp JF, St Sauver J, Sanderson SO, Lindor KD, Feldstein A, Angulo P. The natural history of nonalcoholic fatty liver disease: a population-based cohort study. Gastroenterology. 2005;129:113-21.

Allen AM, Van Houten HK, Sangaralingham LR, Talwalkar JA, McCoy RG. Healthcare Cost and Utilization in Nonalcoholic Fatty Liver Disease: Real-World Data From a Large U.S. Claims Database. Hepatology. 2018;68:6.

Aller R, Izaola O, Gómez S, Tafur C, González G, Berroa E, Mora N, González JM, de Luis DA. Effect of silymarin plus vitamin $\mathrm{E}$ in patients with non-alcoholic fatty liver disease. A randomized clinical pilot study. Eur Rev Med Pharmacol Sci. 2015;19(16):3118-24.

Angulo P. Nonalcoholic fatty liver disease. N. Engl. J. Med. 2002;346:1221-1231.

Bernal-Reyes R, Castro-Narro G, Malé-Velázquez R, Carmona-Sánchez R, González-Huezo MS, GarcíaJuárez I, et al. Consenso Mexicano de la enfermedad por hígado grao no alcohólico. Revista de Gastroenterología de México.2019;84(1):69-99.

Brunt EM, Kleiner DE, Wilson LA, Belt P, Neuschwander-Tetri BA, NASH CRN. The NAS and the Histopathologic Diagnosis in NAFLD: Distinct Clinicopathologic Meanings. Hepatology. 2011;53:810820.

Ceriotti G. Ornithine carbamoyl transferase. Methods of Enzymatic Analysis, edited by Bergmeyer HU, Bergmeyer J, Grassl M. Verlag Chemie; 1983;319-332.

Chen G, Ni Y, Nagata N, Xu L, Ota T. Micronutrient Antioxidants and Nonalcoholic Fatty Liver Disease. Int J Mol Sci. 2016;17(9):1379.

Dowman JK, Tomlinson JW, Newsome PN. Pathogenesis of non-alcoholic fatty liver disease. QJM. 2010;103(2):71-83.

Drake IM, Davies MJ, Mapstone NP, Dixon MF, Schorah CJ, Chalmers DM, et al. Ascorbic acid may protect against human gastric cancer by scavenging mucosal oxygen radicals. Carcinogenesis. 1996;17:559- 
Green LC, Wagner DA, Glogowski J, Skipper PL, Wishnok JS, Tannenbaum SR. Analysis of nitrate, nitrite, and [15N]nitrate in biological fluids. Anal Biochem. 1982;126:131-138.

Gutiérrez-Grobe Y, Juárez-Hernández E, Sánchez-Jiménez BA, Uribe-Ramos MH, Ramos-Ostos MH, Uribe $\mathrm{M}$, et al. Less liver fibrosis in metabolically healthy compared with metabolically unhealthy obese patients with non-alcoholic fatty liver disease. Diabetes Metab. 2017;43(4):332-337.

Gutiérrez-Salinas J, Miranda-Garduño L, Trejo-Izquierdo E, Díaz-Muñoz M, Vidrio S, Morales-González JA, Hernández-Muñoz R. Redox state and energy metabolism during liver regeneration: alterations produced by acute ethanol administration. Biochem Pharmacol. 1999; 58:1831-1839.

Henao-Mejia J, Elinav E, Jin C, Hao L, Mehal WZ, Strowig T, et al. Inflammasome-mediated dysbiosis regulates progression of NAFLD and obesity. Nature. 2012;482:179-85.

Hernández-Muñoz R, Díaz-Muñoz M, and Chagoya de Sánchez V. In vivo and in vitro adenosine stimulation of ethanol oxidation by hepatocytes, and the role of the malate-aspartate shuttle. Biochim Biophys Acta.1987; 930:254-263.

Hoenig MR, Cowin G, Buckley R, McHenery C, Coulthard A. Liver fat percent is associated with metabolic risk factors and the metabolic syndrome in a high-risk vascular cohort. Nutr Metab (Lond). 2010;16;7:50.

Kleiner DE, Brunt EM, Van Natta M, Behling C, Contos MJ, Cummings OW, et al. Design and Validation of a Histological Scoring System for Nonalcoholic Fatty Liver Disease. Hepatology. 2005; 41(6):1312-21.

Leghi GE, Domenici FA, Vannucchi H. Influence of oxidative stress and obesity in patients with nonalcoholic steatohepatitis. Arq Gastroenterol. 2015;52(3):228-33.

Liu J, Tian J, Chaudhry M, Maxwell K, Yan Y, Wang X, et al. Attenuation of Na/K-ATPase Mediated Oxidant Amplification with pNaKtide Ameliorates Experimental Uremic Cardiomyopathy. Scientific reports. 2016; 6: 34592.

Masarone M, Rosato V, Dallio M, Gravina AG, Aglitti A, Loguercio C, et al. Role of Oxidative Stress in Pathophysiology of Nonalcoholic Fatty Liver Disease. Oxidative medicine and cellular longevity. 2018; 2018: 9547613.

National Cholesterol Education Program. Executive summary of the third report of the National Cholesterol Education Program (NCEP) Expert Panel on detection, evaluation, and treatment of high blood cholesterol in adults (Adult Treatment Panel III). J Am Med Assoc. 2001;285:2486-97.

Sodhi K, Puri N, Favero G, Stevens S, Meadows C, Abraham NG, et al. Fructose Mediated Non-Alcoholic Fatty Liver Is Attenuated by H0-1-SIRT1 Module in Murine Hepatocytes and Mice Fed a High Fructose Diet. PloS one. 2015; 10: e0128648. 
Spahis S, Delvin E, Borys JM, Levy E. Oxidative Stress as a Critical Factor in Nonalcoholic Fatty Liver Disease Pathogenesis. Antioxid Redox Signal. 2017;26(10):519-541.

Stienstra R, Stefan N. Tipping the inflammatory balance: inflammasome activation distinguishes metabolically unhealthy from healthy obesity. Diabetologia. 2013;56:2343-6.

Videla LA, Rodrigo R, Araya J, Poniachik J. Oxidative stress and depletion of hepatic long-chain polyunsaturated fatty acids may contribute to nonalcoholic fatty liver disease. Free radical biology \& medicine. 2004;37:1499-507.

Xiong XF, Anderson PM. Purification and properties of ornithine carbamoyl transferase from liver of Squalus acanthias. Arch Biochem Biophys.1989;270:198-207.

Yu NY, Wolfson T, Middleton MS, Hamilton G, Gamst A, Angeles JE, et al. Bone marrow fat content is correlated with hepatic fat content in paediatric non-alcoholic fatty liver disease. Clin Radiol. 2017;72(5):425.e9-425.e14.

\section{Figures}
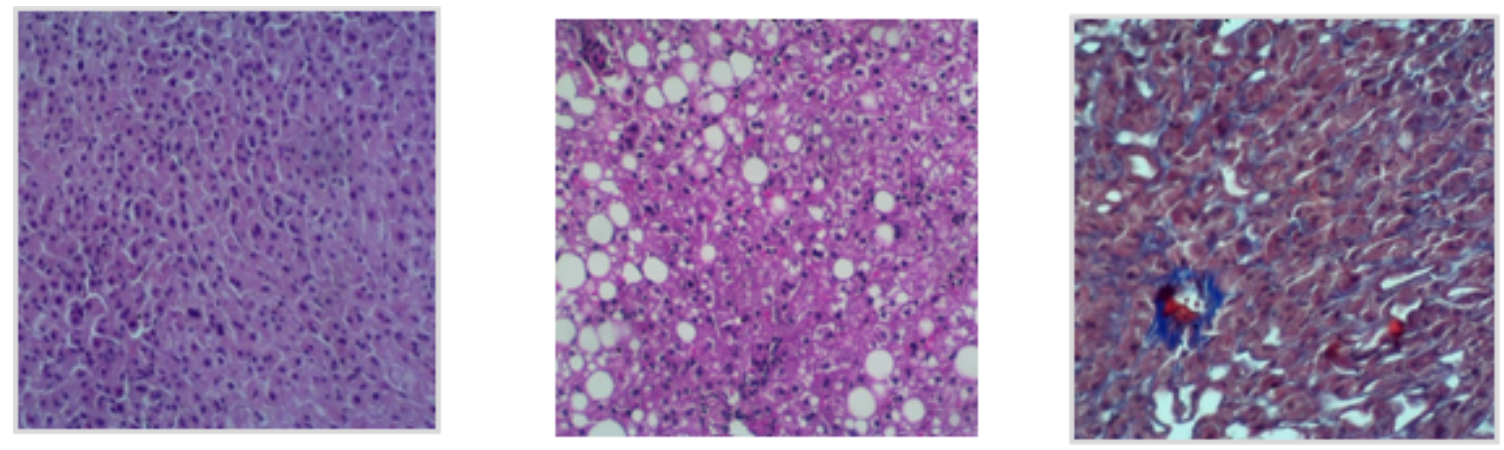

\section{Figure 1}

NAFLD and Metabolic Risk Phenotype. Representative images of liver histology in subgroups according to Metabolic Risk Phenotype, evaluated with Masson's trichrome staining. Left, zero grade of fibrosis with no presence of steatosis. Middle, low grade de fibrosis and mild steatosis. Right, severe stage of fibrosis. 


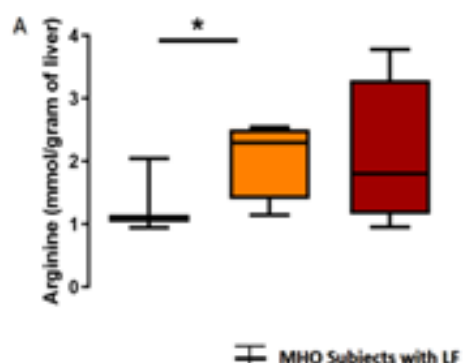

I мно subjects with L5

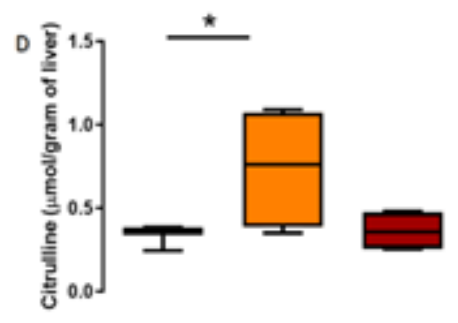

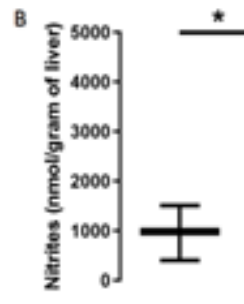
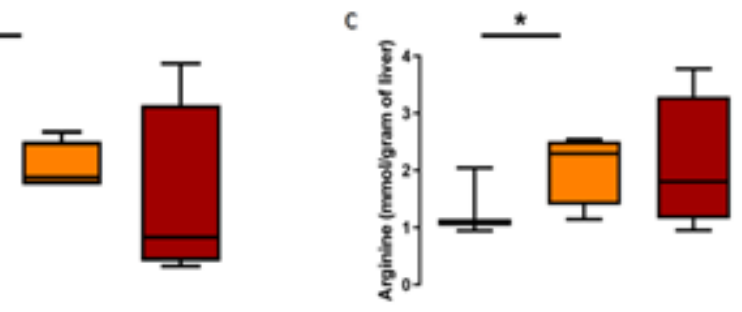

Muo Subjects with HF

- peoss

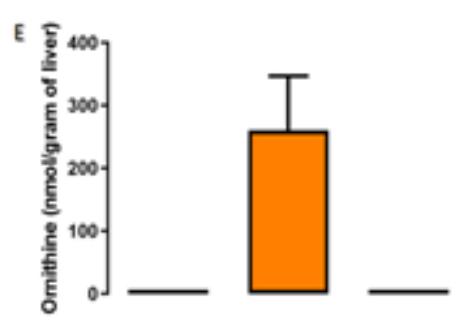

Figure 2

Liver tissue nitrogen metabolic products and metabolic phenotype. Hepatic concentration of nitrogen metabolism products, such as A. ammonium, B. nitrites, C. arginine, D. citrulline and E. ornithine, were compared between groups of NAFLD progression. Abbreviatures: MUO, Metabolically unhealthy obese; MHO, Metabolically healthy obese; LF, low fibrosis; HF, high fibrosis. $\left.{ }^{*}\right) \mathrm{p}<0.05$.

A

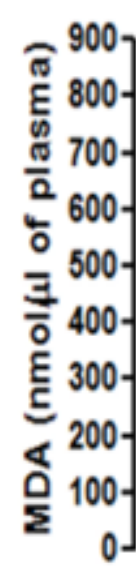

B

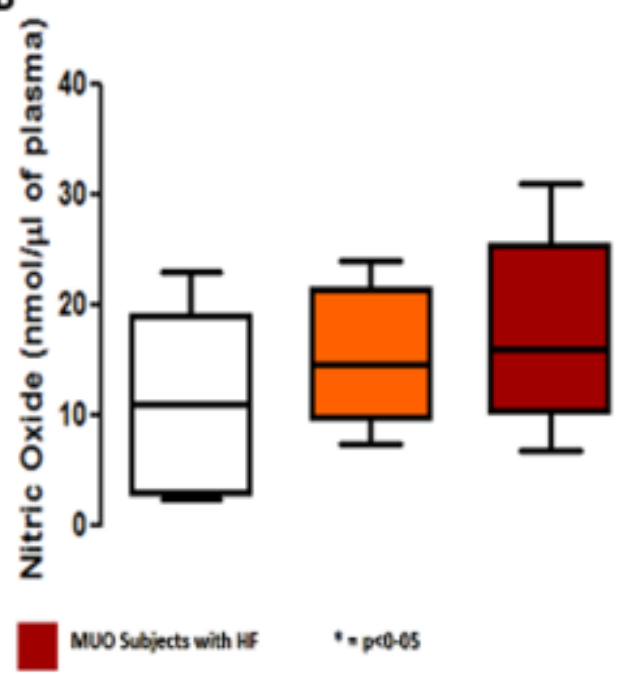

Figure 3

Plasma metabolic products and metabolic phenotype. The levels of MDA and nitric in plasma was assessed from an initial sample of venous blood of $5 \mathrm{ml}$, as a result of several enzymatic kinetics tests. (*) $p<0.04$. The group 2 was not included due to lack of patients. Abbreviatures: MUO, Metabolically unhealthy obese; MHO, Metabolically healthy obese; LF, low fibrosis; HF, high fibrosis. 